\title{
The epidemiology of Type 1 diabetes mellitus is not the same in young adults as in children
}

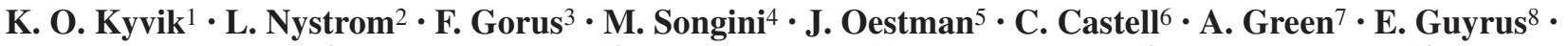 \\ C. Ionescu-Tirgoviste ${ }^{9}$ P. A. McKinney ${ }^{10} \cdot$ D. Michalkova ${ }^{11} \cdot$ R. Ostrauskas ${ }^{12} \cdot$ N. T. Raymond ${ }^{13}$ \\ 1 The Danish Twin Registry, Epidemiology, Institute of Public Health, University of Southern Denmark, Odense C, Denmark \\ 2 Department of Public Health and Clinical Medicine, Umeå University, Umeå, Sweden \\ ${ }^{3}$ The Belgian Diabetes Registry, Brussels, Belgium \\ 4 The Sardinian Diabetes Registry, Diabetes Unit, S. Michele Hospital, Cagliari, Italy \\ ${ }_{5}^{5}$ Centre of Metabolism and Endocrinology, Huddinge University Hospital, Karolinska Institute, Sweden \\ ${ }^{6}$ Advisory Committee on Diabetes in Catalonia, Department of Health and Social Security, Autonomous Government of Catalonia, \\ Spain \\ ${ }^{7}$ Department of Epidemiology and Social Medicine, University of Aarhus, Århus, Denmark \\ ${ }^{8}$ Department of Paediatrics, Faculty of Medicine, University of Pécs, Pécs, Hungary \\ ${ }^{9}$ Institute of Nutrition and Metabolic Diseases "N. Paúlescú", Bucharest, Romania \\ 10 Paediatric Epidemiology Group, University of Leeds, Leeds, UK \\ 11 The Slovak Diabetes Type 1 Registry, University Hospital, Department of Pediatrics, Bratislava, Slovakia \\ 12 Institute of Endocrinology, Kaunas University of Medicine, Kaunas, Lithuania \\ ${ }^{13}$ Department of Epidemiology and Public Health, University of Leicester, Leicester, UK
}

\section{Abstract}

Aims/hypothesis. This prospective study examined the epidemiology of Type 1 diabetes in young adults in Europe.

Methods. We ascertained incident cases of Type 1 diabetes in the 15 to 29 years (both inclusive) age group throughout Europe over a period of 2 years. Diabetes registries in nine countries, in which incidence rates for Type 1 diabetes in the 0 to 14 age group were available, took part. Incidence rates were estimated per 100000 person years and standardised for sex and age. Cumulative incidences per 1000 from birth to age 30 were estimated. Heterogeneity between centres was tested with a Poisson regression model.

Results. A total of 2112 diabetes cases were ascertained in 1996 and 1997, of which 61.4\% were considered to be Type 1 diabetes. Completeness of ascertainment varied from 70 to $90 \%$. Standardised inci- dence varied from 4.8 per 100000 person years to 13.4 per 100000 person years. The male-female ratio was estimated to be one or more, and in the 25 to 29 age group 1.5 or more in all countries. Cumulative incidences for males and females indicate that the former exceeds the latter from age 24 . In the two centres with highest childhood incidence, this applied already from 14 years of age.

Conclusions/interpretation. The incidence of Type 1 diabetes in adults is lower than in children and the range of incidence is also reduced, with a less than threefold variation in adults, against an eightfold variation in children. There is a male excess in incidence, especially in the age group 25 to 29 years. [Diabetologia (2004) 47:377-384]

Keywords Type 1 diabetes - Incidence - Cumulative incidence $\cdot$ Epidemiology $\cdot$ Adults
Received: 17 March 2003 / Revised: 5 December 2003

Published online: 5 February 2004

C Springer-Verlag 2004

K. O. Kyvik (®)

The Danish Twin Registry, Epidemiology, Institute of Public Health, University of Southern Denmark, Sdr. Boulevard 23 A, 5000 Odense C, Denmark

E-mail:kkyvik@health.sdu.dk

Abbreviations: EURODIAB TIGER, European Diabetes: Type I Genetic Epidemiology Resource
Type 1 (insulin-dependent) diabetes mellitus is a common chronic disease of childhood, and presents with acute, sometimes life-threatening, symptomatic hyperglycaemia. Children with diabetes present to paediatric services and are highly visible within the health system. This is not the case in patients who are 15 to 29 years of age; symptoms are typically less acute, and both gestational and Type 2 diabetes are increasingly prevalent in this age group, compared with children $[1,2]$. Adolescent and young adult patients are 
treated in diabetes units, general medical or obstetrical units, or by general practitioners. Consequently ascertainment and classification of Type 1 diabetes are more difficult than in children, and standardised comparisons between age groups and between areas with differing incidences of Type 1 diabetes in childhood have not been carried out.

Type 1 diabetes is a multifactorial disease in which environmental risk factors trigger an immune-mediated destruction of the pancreatic beta cells in genetically susceptible individuals [3]. Studies of the epidemiology of Type 1 diabetes in different populations can provide important clues about putative environmental risk factors. The incidence of the disease in children up to 15 years of age is well documented by two multi-centre studies $[4,5,6,7]$, with incidence varying from less than 1 per 100000 person years in Venezuela and China to more than 35 per 100000 person years in Finland and Sardinia. Furthermore, recent studies suggest a higher rate of increase in disease among children younger than 5 years than in children between 5 and 15 years of age $[8,9,10,11]$, although other studies have not observed a differential rise in incidence $[12,13,14]$.

Although Type 1 diabetes has traditionally been considered a disease of childhood, the majority of cases present in adult life [15]. Adult cases are characterised by a reduced frequency of HLA susceptibility haplotypes, as compared with children [1, 16, 17], and overall have less acute symptoms than children [1]. Male predominance is a further, unexplained feature of Type 1 diabetes in young adults $[18,19,20,21,22$, $23,24,25,26,27]$. This is in marked contrast to most other common immune-mediated conditions, which are typified by female preponderance [28].

The epidemiology of Type 1 diabetes in adults is less well characterised than in children of 0 to 14 years of age, especially with regard to international comparisons of incidence rates and to differences in incidence between the sexes. Here we report on a collaborative research project, which has studied the incidence of Type 1 diabetes mellitus in the 15- to 29-year age group in European regions known to represent high, intermediate and low childhood incidence areas to investigate the geographical distribution by sex and age of Type 1 diabetes mellitus with onset in young adulthood.

\section{Subjects and methods}

Centres participating in the study. European centres were eligible for participation on the basis of the following requirements: first, they had to be able to identify all new cases of diabetes mellitus or all new cases of Type 1 diabetes mellitus in the 15- to 29- year age group, as reported by treating physicians in a geographically well-defined population with reliable demographic data. Second, they had to be able to validate the completeness of registration by means of one or more independent secondary source of ascertainment [29].
A total of nine centres are taking part in the concerted action and results are complete for 1996 and 1997. The participating centres represent geographical areas known from European Diabetes: Type I Genetic Epidemiology Resource (EURODIAB TIGER) to be areas with high (Sardinia and Sweden), intermediate (England, Antwerp in Belgium and Catalonia in Spain) and low (Lithuania, Bucharest in Romania and Slovakia) Type 1 diabetes incidence in children. In Sweden only childhood incidence data from Stockholm were used for the EURODIAB studies of childhood Type 1 diabetes, and in Yorkshire, England only the western part of the area (West Yorkshire) took part in the study of young adults. Thus our comparison is also based on this.

Data sources and classification of disease. In all centres, direct notification from hospitals, diabetologists and/or general practitioners constituted the primary data source. Prescriptions, diabetes associations, summer camps, registries of social benefits and general practitioners were used as secondary sources

Classification of diabetes was based on the diagnosis made by the diabetologist and the date of the first insulin injection was used as date of diagnosis of Type 1 diabetes.

Statistical analysis. Population data from each local study centre's geographically defined area were used to calculate ageand sex-specific incidences. Incidence rates per 100000 person years at risk and $95 \%$ CI were calculated. The incidence rates were standardised for sex and age, with the pooled data used as the standard. The $95 \%$ CI were estimated using an approximated Poisson distribution as described previously [30]. Incidence data from 1996 and 1997 in children of 0 to 14 years of age were provided by EURODIAB TIGER, details of which have been previously published $[4,6]$.

Incidence rates within centres, e.g. children versus adults, males versus females and males versus females in the two age categories, were compared with chi square and MantelHaenszel chi square tests and 95\% CI for the incidence rate ratios calculated. Differences between centres were tested using a Poisson regression model. Cumulative incidence per 1000 was calculated and sex-specific cumulative incidence was analysed in groups based on childhood incidence in areas with low, intermediate and high childhood incidence. Trends in cumulative incidences were assessed by linear and log-linear regression models. The curves were compared using multivariate general linear regression models. We considered $p$ values below 0.05 to be statistically significant. The software packages Excel, SPSS (SPSS, Chicago, Ill., USA) and Stata version 7 (StataCorp, College Station, Tex., USA) were used.

Ethics. The study was carried out in accordance with the Helsinki Declaration as revised in 2000. All local study centres fulfilled the ethical requirements of their national legislation, and had permission from their local scientific and ethics commission, as well as from their national data protection agencies where applicable. Only anonymous data were submitted to the central co-ordinating office in Denmark and this was approved by The Danish Data Protection Board.

\section{Results}

Of the 2112 cases of diabetes that were ascertained in 1996 and 1997 in the 15 to 29 age group, 1296 (61.4\%) were classified as Type 1 diabetes cases by referring doctors (Table 1). Completeness of ascertainment within the local study centres varied from 70 to $90 \%$. 
Table 1. Ascertainment of Type 1 diabetes cases in young adults (men and women) by study centre for 1996 and 1997

\begin{tabular}{lcrr}
\hline Centre & Completeness of ascertainment (95\% CI) & Number of cases, men & Number of cases, women \\
\hline Belgium (Antwerp region) & $90 \%(86-93 \%)$ & 17 & 16 \\
Italy (Sardinia) & $70 \%(58-84 \%)$ & 71 & 33 \\
Lithuania & $90 \%(79-100 \%)$ & 81 & 51 \\
Romania (Bucharest area) & $100 \%$ a & 45 & 32 \\
Spain (Catalonia) & $90 \%(87-93 \%)$ & 200 & 116 \\
UK (Leicestershire) & $90 \%(84-98 \%)$ & 29 & 22 \\
UK (West Yorkshire) & $98 \%(96-99 \%)$ & 67 & 35 \\
Sweden & $85 \%$ b & 238 & 127 \\
Slovakia & $80 \%(71-89 \%)$ & 77 & 49
\end{tabular}

a According to the Bucharest centralised medical system for diabetes care, all diabetic patients from Bucharest are registered in the Diabetes Centre and thus in the registry. Free in- sulin is distributed only by the pharmacy belonging to this Centre.

$\mathrm{b}$ Average of several studies of level of ascertainment

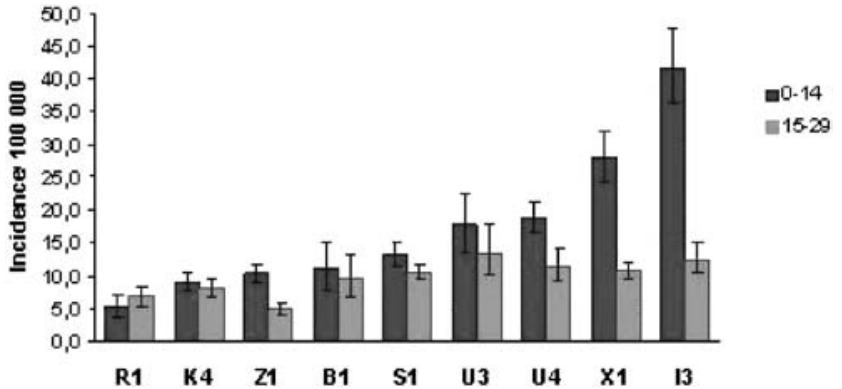

Fig. 1. Standardised incidences with $95 \%$ CI of Type 1 diabetes in children and young adults by study area. Results are arranged according to childhood incidence. Black bars: 0-14 years (children); shaded bars: 15-29 years (young adults). R1, Romania (Bucharest area); K4, Lithuania; Z1, Slovakia; B1, Belgium (Antwerp area); S1, Spain (Catalonia); U3, UK (Leicestershire); U4, UK (West Yorkshire); X1, Sweden; I3, Italy (Sardinia)

The total incidence for 1996 to 1997 in young adults varied from 4.8 per 100000 person years in Slovakia to 13.4 per 100000 in Leicestershire, England. Age- and sex-specific rates are shown in Table 2 and standardised rates are illustrated in Fig. 1. In Bucharest, Romania the incidence rates in adults was higher than in children, although this was not statistically significant $(p=0.9)$. In all the other centres incidence was lower in young adults than in children. This was only statistically significant in Catalonia, Spain $(p=0.05)$, Yorkshire, England $(p<0.001)$, Sardinia, Italy $(p<0.001)$ and Sweden $(p<0.005)$. In the centres with a high incidence in children the incidence in young adults was approximately one-third of that value or less, the incidence rate ratios for Sardinia and Sweden being 3.3 and 2.6. Heterogeneity among centres was established (Poisson regression LR chi square, $6 d f=111.33$; H0: no difference among centres: $p<0.001)$.

The cumulative incidence of Type 1 diabetes mellitus before age 30 (Fig. 2) fitted a linear curve in all centres, except Sweden and Sardinia which had a logarithmic curve. The curves from all centres showed
Cumulative risk in all centers 1996-1997

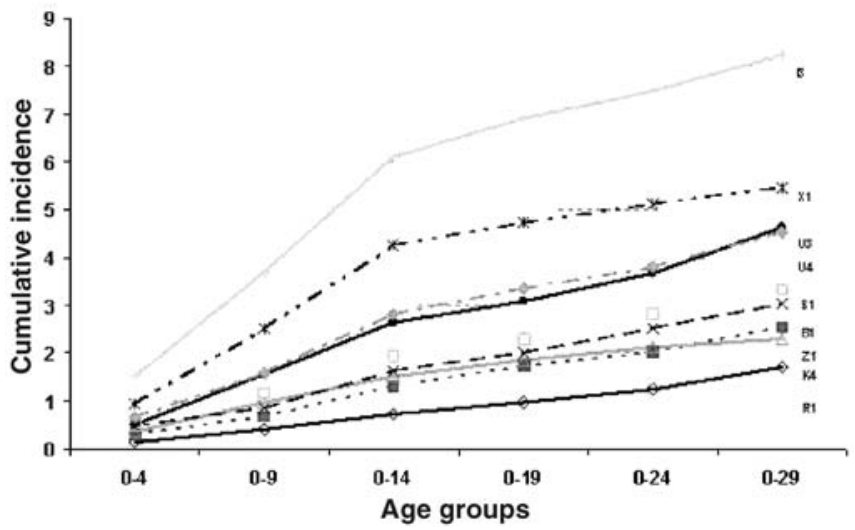

Fig. 2. Cumulative incidence of Type 1 diabetes from 0 to 30 years

significant trends, but multivariate analysis did not result in significant differences between the slopes of the centres $(p>0.05)$. The cumulative incidence in males and females (Fig. 3a-c) when centres were grouped according to low, intermediate and high levels of incidence of childhood diabetes shows a tendency for cumulative incidence in males to exceed that in females from the age of 25 years. For the two centres with the highest childhood incidence, this applied already from age 15 .

With the exception of Slovakia, where there was a higher incidence in male children than in female children $(p<0.005)$, incidence rates in children younger than 15 years of age did not vary according to sex. In all centres the point estimates of the male to female incidence rate ratio were greater than one in young adults. The sex-specific incidence rates in the total age group (15-29 years) were statistically different in all centres apart from Antwerp (Belgium) and Leicestershire (England). In the 25 to 29 age group all ratios exceeded 1.5 (Fig. 4) and differed significantly from one in all centres except Antwerp and Leicestershire (Table 2). The stratified analysis of incidence rates in 
K. O. Kyvik et al.:

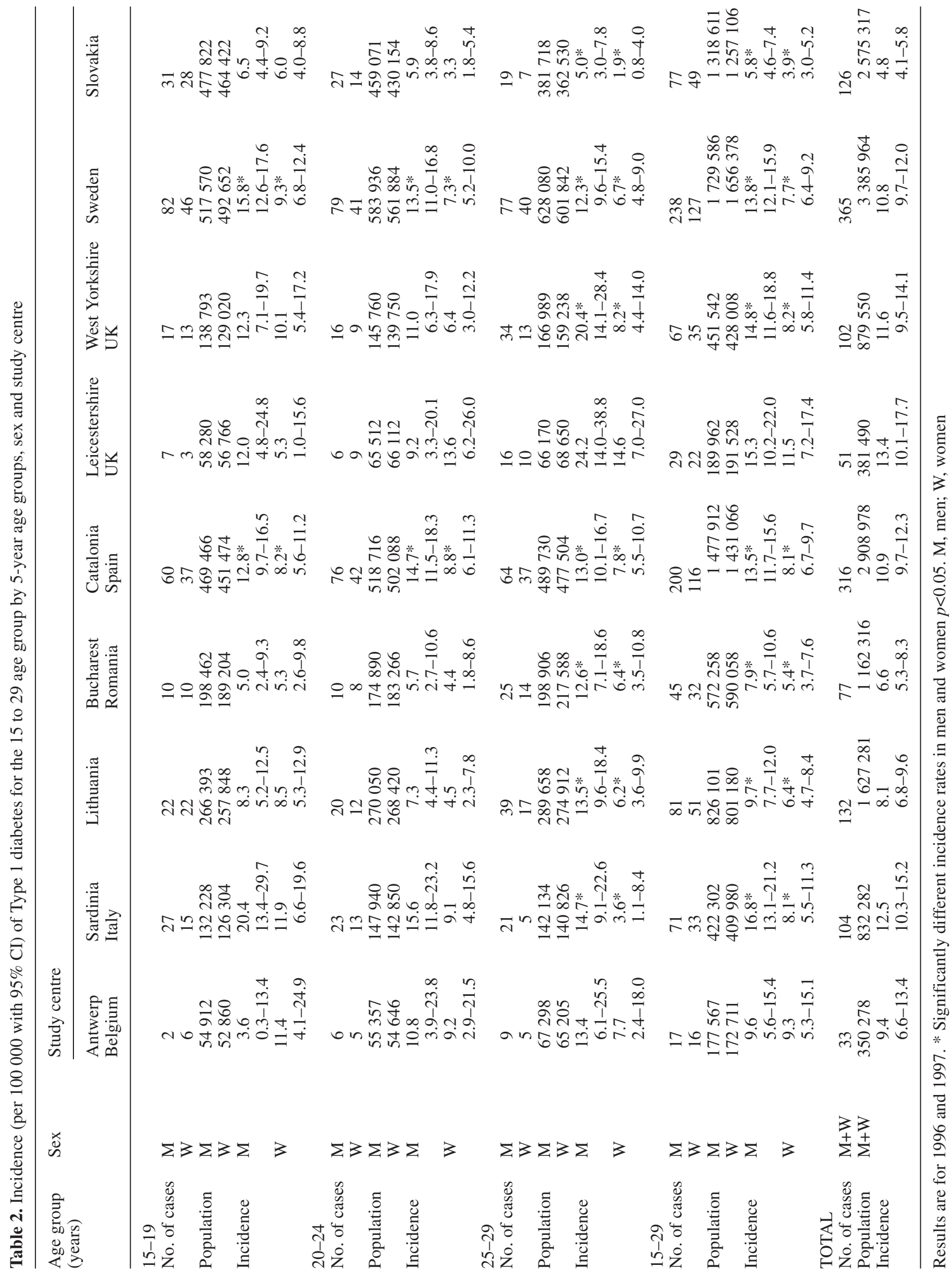


a Cumulative risk of Type 1 diabetes in areas with low childhood incidence

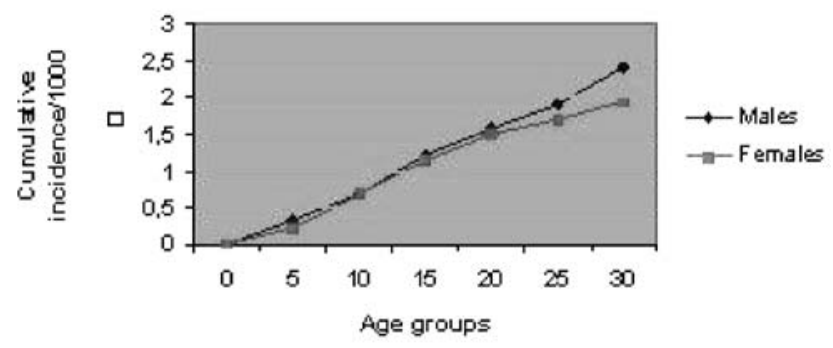

b Cumulative risk of Type 1 diabetes in areas with intermediate childhood incidence

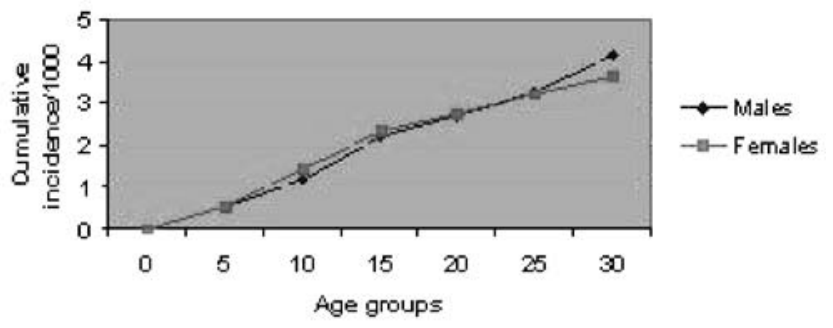

C Cumulative risk of Type 1 diabetes in areas with high childhood incidence

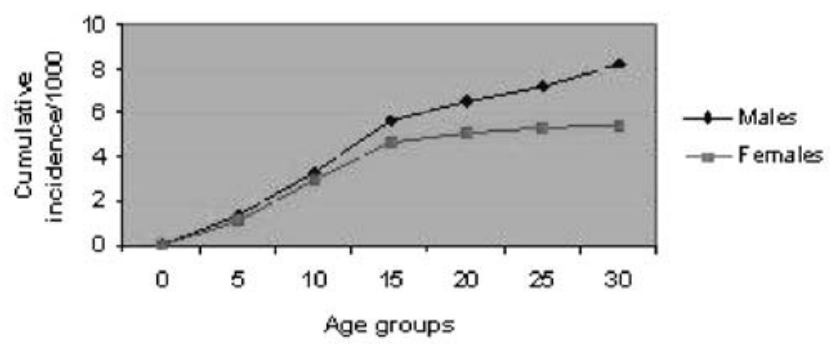

Fig. 3a-c. Sex-specific cumulative incidence of Type 1 diabetes for study areas with low (a), intermediate (b) and high (c) childhood incidence

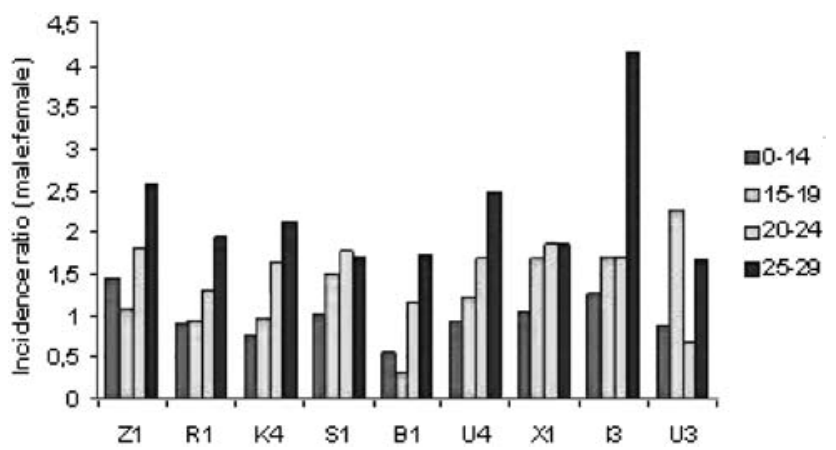

Fig. 4. Male to female ratio of Type 1 diabetes incidence by age groups and study areas. Dark grey bars: 0-14 years; light grey bars: 15-19 years; open bars: 20-24 years; black bars: 25-29 years. R1, Romania (Bucharest area); K4, Lithuania; Z1, Slovakia; B1, Belgium (Antwerp area); S1, Spain (Catalonia); U3, UK (Leicestershire); U4, UK (West Yorkshire); X1, Sweden; I3, Italy (Sardinia) male children and female children (0-14 years of age) compared with young adults (15-29 years of age) showed significant differences between the two groups in Slovakia $(p<0.0001)$, Catalonia $(p<0.0001)$ and in both centres with high incidence of Type 1 diabetes in children, i.e. Sweden $(p<0.0001)$ and Sardinia $(p<0.0001)$.

\section{Discussion}

The incidence of Type 1 diabetes in children varies more than tenfold across Europe and 350-fold between Finland and China [4, 7]. We selected study areas with a childhood incidence ranging from 5.3 per 100000 person years (Bucharest) to 41.6 per 100000 person years (Sardinia), and showed that there is far less variation in incidence in young adults from the same regions. In all centres but Bucharest the incidence in young adults was lower than in children, and the incidence rate ratios in Sardinia and Sweden, the countries with the highest childhood incidence, were over 2.5 .

In all nine centres the estimated male to female ratio of Type 1 diabetes incidence was greater than one in the 15 to 29 age group and greater than in children. In the age group 25 to 29 years the male to female incidence rate ratio was equal to or greater than 1.5 in all centres, although this was not statistically significant in Antwerp and Leicestershire, the two centres with the smallest study populations [21, 31]. However, the Belgian Diabetes Registry has shown a significant difference between incidence rates in men and women $(p<0.001)$ in the age range 15 to 39 years [21, 32].

A potential problem with this study is the completeness of ascertainment, which was lower than in the childhood studies $[4,6,7]$. This is a reflection of the difficulties encountered when trying to ascertain young adults, who are less visible in the health care system, while diabetes in this age group is more heterogeneous and classification more complex. Patients in this age group are treated in diverse settings, making capture from multiple-ascertainment sources necessary for completeness. They are also much more mobile. Under these circumstances the completeness of ascertainment obtained in our study can be regarded as satisfactory, as it was comparable to previous findings [7].

Some of the centres participating in this EU-project ascertain few cases from limited geographical areas, and this can result in loss of power to detect differences. In Belgium a national diabetes registry exists, but achieves only $40 \%$ ascertainment. A comparison between the Antwerp registry with $92 \%$ ascertainment and the national Belgian registry with $40 \%$ ascertainment showed that demographic, clinical and biological data did not differ in the two registries, indicating 
that the registry with $40 \%$ ascertainment was nevertheless representative [21]. However, we chose to use the smaller Antwerp registry for this EU project because high ascertainment is more important than representability of cases, when it comes to ensuring maximum reliability of incidence rate estimates. A longer observation period will thus be necessary to obtain better estimates.

Another potential problem in this study is the difficulties involved in classifying type of diabetes in patients older than 14 years of age. HLA-types and immunological markers have not been used for classification, but the treating physicians did of course have clinical information. The same information is in the data sheets used for report at diagnosis. An earlier study on the difficulties of classifying type of diabetes showed that more cases are initially classified as Type 1 diabetes and later changed to Type 2 diabetes than the other way around [33]. The estimates of incidence rates in this study could therefore be regarded as conservative.

The strength of our study is that incidence data were ascertained in the same standardised way in centres throughout Europe for which well-validated incidence rates of childhood diabetes were available.

The difference in incidence between populations was much less marked in the 15 to 29 than in the 0 to 14 age groups. There was, however, heterogeneity between countries. Regional European differences with regard to socio-economic standards and maternal and child health could possibly contribute to these differences. A comparison of the cumulative rate of Type 1 diabetes up to age 30 within the countries studied shows that the two countries with the highest childhood incidence both had a slow-down of the rate of increase after age 15 , whereas the remaining countries had a continued linear trend. Our results indicate that caution should be exercised when extrapolating regional differences in incidence of childhood onset Type 1 diabetes to differences in lifelong risk for the disease. To decide whether the higher and increasing incidence rates of childhood Type 1 diabetes in certain countries are due to earlier disease presentation $[1,8$, $9,10]$ or to an overall increased lifelong risk when compared to countries with lower incidence rates of childhood Type 1 diabetes, it would be necessary to compare incidence rates across countries over even larger age-ranges and longer observation periods.

A number of studies have reported a strong male preponderance in the incidence of Type 1 diabetes in adults $[18,19,20,21,22,23,24,25,26,27]$. In this respect young adults differ from children, for overall sex-specific differences in children are slight, although high-incidence populations tend to show male preponderance, and low-incidence populations (mostly non-European) show female preponderance [34]. We therefore considered sex in relation to childhood incidence of Type 1 diabetes in our populations. In fact, all participating centres had a male excess in incidence rates, although this was not statistically significant in Antwerp and Leicestershire. From the centres with the highest childhood incidence Sweden had a clear male incidence excess as early as the age of 14 years. In the areas with intermediate childhoodonset diabetes incidence Catalonia had the same male excess pattern, whereas data from Sardinia, the English centres and the low-incidence areas Romania, Slovakia and Lithuania only showed a male excess from the age of 25 years. Studies from Belgium with a longer follow-up period have also found a male excess as described here [21, 34].

The difference in incidence rates between the sexes could be due to factors operating in males, in females, or in both. One possible explanation of the sex-difference in incidences is under- ascertainment of women with Type 1 diabetes who are diagnosed with gestational diabetes, because these were excluded from the Insulin-dependent Diabetes in young Adults study, although primary ascertainment sources in each locality would be expected to register those with Type 1 diabetes after a delay. Most other autoimmune diseases, e.g. autoimmune thyroid diseases, rheumatoid arthritis and multiple sclerosis, have a high female to male ratio, and it is not clear why Type 1 diabetes mellitus shows the opposite picture [28]. The reasons for the difference are not clear, but possibly related to oestrogen secretion in women or to a higher metabolic burden due to android fat distribution. Obesity and insulin resistance could precipitate post-pubertal clinical onset of a slowly progressive immune-mediated diabetes [31].

A study of English, American and Sardinian patients with Type 1 diabetes showed that the male to female excess was restricted to HLA-DR3 positive subjects lacking $D R 4$, thus providing evidence of a linkage between chromosome Xp and diabetes [35]. A Belgian study failed to confirm the finding of HLA restriction with regard to the male to female excess in young adulthood, but did not completely rule out the suggestion of an interaction between an X-linked genetic factor and high-risk HLA types as a route to diabetes [31].

Although Type 1 diabetes is commonly considered a disease of children, only a minority of cases actually present in childhood [1, 2, 17]. We still know remarkably little about the "silent majority" that develop the condition in adult life. Our study, an international comparison of incidence in children and young adults, has shown that the incidence of Type 1 diabetes in young adults in Europe is lower than in children in most areas, and that the range of incidence is also reduced. We have also confirmed a consistent male excess of cases in this age group, which is most marked over the age of 25 and which remains unexplained, standing in marked contrast to the female excess characteristic of most common immune-mediated condi- 
tions. Our understanding of Type 1 diabetes is likely to remain biased and incomplete as long as we focus upon its childhood variant, which is only the tip of the iceberg.

Acknowledgements. This work was financially supported by the European Commission, BIOMED IV programme, Contract number: BMH4-CT98-3369 (DG12-SSMI). We hereby express sincere thanks to Dr A. Goday, Spain, Dr B. Urbonaite, Lithuania, Professor G. Dahlquist, Sweden, Professor G. Soltezs, Hungary, Dr I. Weets, Belgium, J. Jones, UK, and the Sardinian collaborative group for the Epidemiology of Type 1 Diabetes. Registered nurse M. Skott and Bachelor of Business Languages J. Duerlund Jensen are thanked for expert technical help.

\section{References}

1. Karjalainen J, Salmela P, Ilonen J, Surcel HM, Knip M (1989) A comparison of childhood and adult type I diabetes mellitus. N Engl J Med 320:881-886

2. Krolewski AS, Warram JH, Rand LI, Kahn CR (1987) Epidemiologic approach to the etiology of type I diabetes mellitus and its complications. N Engl J Med 317:1390-1398

3. Cordell HJ, Todd JA (1995) Multifactorial inheritance in type 1 diabetes. Trends Genet 11:499-504

4. Green A, Gale EA, Patterson CC (1992) Incidence of childhood-onset insulin-dependent diabetes mellitus: the EURODIAB ACE Study. Lancet 339:905-909

5. Green A, Patterson C (2001) Trends in the incidence of childhood-onset diabetes in Europe 1989-1998. The EURODIAB TIGER Study Group. Diabetologia 44 [Suppl]:B3-B8

6. EURODIAB ACE Study Group (2000) Variation and trends in incidence of childhood diabetes in Europe. EURODIAB ACE Study Group. Lancet 355:873-876

7. Karvonen M, Viik-Kajander M, Moltchanova E, Libman I, La Porte R, Tuomilehto J (2000) Incidence of childhood type 1 diabetes worldwide. The Diabetes Mondiale (DiaMond) Project Group. Diabetes Care 23:1516-1526

8. Dahlquist G, Mustonen L (2000) Analysis of 20 years of prospective registration of childhood onset diabetes time trends and birth cohort effects. Swedish Childhood Diabetes Study Group. Acta Paediatr 89:1231-1237

9. Jarosz-Chobot P, Otto-Buczkowska E, Koehler E, Matlakiewicz E, Green A (2000) Increased trend of type 1 diabetes mellitus in children's population (0-14 years) in Upper Silesia region (Poland). Med Sci Monit 6:573-580

10. Gardner SG, Bingley PJ, Sawtell PA, Weeks S, Gale EA (1997) Rising incidence of insulin dependent diabetes in children aged under 5 years in the Oxford region: time trend analysis. The Bart's-Oxford Study Group. BMJ 315:713-717

11. Podar T, Solntsev A, Karvonen M et al. (2001) Increasing incidence of childhood-onset type 1 diabetes in 3 Baltic countries and Finland 1983-1998. Diabetologia 44 [Suppl]:B17-B20

12. Rangasami JJ, Greenwood DC, McSporran B, Smail PJ, Patterson CC, Waugh NR (1998) Childhood insulin dependent diabetes: Oxford may not be representative. BMJ 316:391-392

13. Rangasami JJ, Greenwood DC, McSporran B, Smail PJ, Patterson CC, Waugh NR (1997) Rising incidence of type 1 diabetes in Scottish children, 1984-93. The Scottish Study Group for the Care of Young Diabetics. Arch Dis Child 77:210-213
14. Feltbower RG, McKinney PA, Parslow RC, Stephenson CR, Bodansky HJ (2003) Type 1 diabetes in Yorkshire, UK: time trends in 0-14 and 15-29-year-olds, age at onset and age-period-cohort modelling. Diabet Med 20:437-441

15. Lorenzen T, Pociot F, Hougaard P, Nerup J (1994) LongTerm risk of IDDM in first-degree relatives of patients with IDDM. Diabetologia 37:321-327

16. Gorus FK, Vandewalle CL, Dorchy H, Crombrugge P van, Schuit FC, Pipeleers DG (1994) Influence of age on the associations among insulin autoantibodies, islet cell antibodies, and HLA DAQ1*0301-DQB1*0302 in siblings of patients with type 1 (insulin-dependent) diabetes mellitus. Belgian Diabetes Registry. J Clin Endocrinol Metab 78:1172-1178

17. Gorus FK, Anselmo J, Keymeulen B (1993) Type 1 (insulindependent) diabetes mellitus: an autoimmune, predictable and preventable disease? Lessons from national registries and new challenges to clinical biology. Acta Clin Belg 48:96-108

18. Blohme G, Nystrom L, Arnqvist HJ et al. (1992) Male predominance of type 1 (insulin-dependent) diabetes mellitus in young adults: results from a 5-year prospective nationwide study of the 15-34-year age group in Sweden. Diabetologia 35:56-62

19. Bruno G, Merletti F, Vuolo A, Pisu E, Giorio M, Pagano G (1993) Sex differences in incidence of IDDM in age-group 15-29 yr. Higher risk in males in Province of Turin, Italy. Diabetes Care 16:133-136

20. Nystrom L, Dahlquist G, Ostman J et al. (1992) Risk of developing insulin-dependent diabetes mellitus (IDDM) before 35 years of age: indications of climatological determinants for age at onset. Int J Epidemiol 21:352-358

21. Vandewalle CL, Coeckelberghs MI, De Leeuw IH et al. (1997) Epidemiology, clinical aspects, and biology of IDDM patients under age 40 years. Comparison of data from Antwerp with complete ascertainment with data from Belgium with $40 \%$ ascertainment. The Belgian Diabetes Registry. Diabetes Care 20:1556-1561

22. Roglic G, Pavlic Renar I, Sestan-Crnek S et al. (1995) Incidence of IDDM during 1988-1992 in Zagreb, Croatia. Diabetologia 38:550-554

23. Goday A, Castell C, Tresserras R, Canela J, Taberner JL, Lloveras G (1992) Incidence of type 1 (insulin-dependent) diabetes mellitus in Catalonia, Spain. The Catalan Epidemiology Diabetes Study Group. Diabetologia 35:267-271

24. Christau B, Kromann H, Christy M, Andersen OO, Nerup J (1979) Incidence of insulin-dependent diabetes mellitus (0-29 years at onset) in Denmark. Acta Med Scand [Suppl] 624:54-60

25. Joner G, Sovik O (1991) The incidence of type 1 (insulindependent) diabetes mellitus 15-29 years in Norway 1978-1982. Diabetologia 34:271-274

26. Bruno G, Merletti F, Pisu E, Pastore G, Marengo C, Pagano G (1990) Incidence of IDDM during 1984-1986 in population aged less than 30 yr. Residents of Turin, Italy. Diabetes Care 13:1051-1056

27. Muntoni S, Songini M (1992) High incidence rate of IDDM in Sardinia. Sardinian Collaborative Group for Epidemiology of IDDM. Diabetes Care 15:1317-1322

28. Gale EA, Gillespie KM (2001) Diabetes and gender. Diabetologia 44:3-15

29. Hook EB, Regal RR (1992) The value of capture-recapture methods even for apparent exhaustive surveys. The need for adjustment for source of ascertainment intersection in attempted complete prevalence studies. Am J Epidemiol 135:1060-1067

30. Diem K, Lentner C (1970) Documenta Geigy Scientific Tables, 7th edn. Geigy, Basle 
31. Weets I, Autreve J van, Auwera BJ van der et al. (2001) Male-to-female excess in diabetes diagnosed in early adulthood is not specific for the immune-mediated form nor is it HLA-DQ restricted: possible relation to increased body mass index. Diabetologia 44:40-47

32. Gorus FK (1997) Diabetes registries and early biological markers of insulin-dependent diabetes mellitus. Belgian Diabetes Registry. Diabetes Metab Rev 13:247-274

33. Arnqvist HJ, Littorin B, Nystrom L et al. (1993) Difficulties in classifying diabetes at presentation in the young adult. Diabet Med 10:606-613
34. Karvonen M, Pitkaniemi M, Pitkaniemi J, Kohtamaki K, Tajima N, Tuomilehto J (1997) Sex difference in the incidence of insulin-dependent diabetes mellitus: an analysis of the recent epidemiological data. World Health Organization DIAMOND Project Group. Diabetes Metab Rev 13:275291

35. Cucca F, Goy JV, Kawaguchi Y et al. (1998) A malefemale bias in type 1 diabetes and linkage to chromosome $\mathrm{Xp}$ in MHC HLA-DR3-positive patients. Nat Genet 19:301-302 